\title{
Low Power Wide Area IoT Networks: Reliability Analysis in Coexisting Scenarios
}

\author{
Meysam Masoudi, Amin Azari, and Cicek Cavdar
}

\begin{abstract}
Low-power wide-area networks (LPWANs) are emerging technologies aiming at serving sporadic IoT traffic. While reliability of LPWANs in single-technology deployment has been well investigated, there is lack of research on performance evaluation in coexisting scenarios. To tackle this problem, we start by modeling statistics of aggregated interference from asynchronous sources over shared radio resources. Then, using stochastic geometry, we derive the closed-form statistics of reliability, mean success probability, and the mean local delay, defined as the mean time (in numbers of attempts) until a packet is successfully received by the nearest access point. We further investigate the interplay between traffic-load, access points density, and quality of communications in coexistence scenarios. Then, we derive the communications parameters regions, in terms of transmission power, access point density, and time activity factor, for which, the mean local delay is finite. The numerical and simulation results also corroborate the analysis.
\end{abstract}

Index Terms-IoT Communications, LPWAN, Grant-free Access, Mean Local Delay, Meta Distribution.

\section{INTRODUCTION}

Enabling large-scale Internet of Things (IoT) communications, which is a key driver of 5th generation of wireless access networks and beyond (5GB/6G), relies heavily on introduction of scalable, cost-efficient, low-power communications protocols/technologies [1]. Based on the range and spectrum of communications, the state-of-the-art proposed technologies for enabling IoT communications can be categorized into: 1) short-range solutions, e.g., WiFi, Bluetooth; 2) long-range solutions over licensed spectrum, e.g., LTE-M, NB-IoT; and 3) long-range solutions over unlicensed spectrum, e.g., LoRA, SigFox. The last two categories, also known as low power wide area networks (LPWANs), aiming at providing simplified radio access protocols for low-cost low-power IoT devices. Especially, the last category leverages grant-free access which simplifies the resource reservation procedure for battery constrained IoT devices at the cost of degrading the reliability of communications.

The literature on the reliability of LPWANs' communications is mainly focusing on single technology deployment, i.e. when the characteristics of communication; including transmit power, size of packets, and packet generation rates; are the same across different devices [1-5]. The authors in [2] propose a framework for modeling the performance of a single LPWAN gateway and investigate the scalability of such networks. Reliability of communication without prior resource reservation, as implemented in LPWANs, has been studied in [3], by assuming channel inversion power control for IoT devices,

M. Masoudi and C. Cavdar are with the School of Electrical Engineering and Computer Science, KTH Royal Institute of Technology, Stockholm, Sweden, (e-mail:\{masoudi,cavdar\}@kth.se).

A. Azari is with the Ericsson Research, Stockholm, Sweden, (email:amin.azari@ericsson.com). which is hardly met in practical scenarios. For the same setup, reliability of communications has been investigated in [4], where simplified expressions for outage probability and throughput have been derived. In [1], the reliability and latency performance of LPWAN has been investigated taking into account the regulations on time activity of devices. In [5], the reliability of LPWAN links has been investigated for additive white Gaussian noise and Rayleigh fading channels without considering interference in the network. Recent studies show that in practical scenarios, e.g. a hospital complex, multiple technologies coexist in the same service area over shared radio resources without any control over the resource utilization, and hence, may suffer significantly from the intertechnology interference [6]. In [7], energy efficiency and coverage probability of multiple LoRa networks have been investigated (intra-technology interference), while coexistence with other IoT technologies is missing.

Besides the lack of research on inter-technology interference for LPWANs, the prior arts mainly define reliability of IoT communications [4-8] in terms of average success probability conditioned on the underlying point processes describing distribution of devices. For example, in [8], we have presented early results on performance evaluation for co-existing IoT technologies in terms of average success probability in uplink communications. This performance measure provides limited information about the performance since the information about bottleneck links are lost when it is averaged over the whole network. In other words, this metric does not provide an indication of percentage of devices that their data will be discarded in the service area because of unsuccessful attempts before data expiration. To tackle this issue, stochastic geometry can be used to derive meta distribution of reliability or success probability. Unlike the transmission success probability which depends only on the SINR threshold, meta distribution is a function of both the SINR threshold and the required reliability threshold and it is defined as the complementary cumulative distribution function of conditional success probability with respect to the point processes and activities of devices [9]. For a given SINR threshold, and due to the ergodicity of the point processes, it provides insights on the fraction of users in the network with the desired link reliability. Despite high average reliability across the network, in LPWANs there might be some users in the network who cannot complete their transmission in a finite time as a result of interference between coexisting technologies. It is important to investigate under what conditions this might happen. The studies in $[10,11]$, provided an efficient way to calculate the interference and meta distribution for cellular networks and D2D communications. In cellular networks (including D2D communications [12]), interference is managed to some extent leveraging advanced 
power control and resource allocation/scheduling algorithms. However, in LPWANs, interference originates from a massive number of asynchronous devices with random hopping over time/frequency resources and results in partially overlapping transmissions. Hence, the results of [10-12] are not applicable to LPWANs. Similar analysis for LPWANs can help service providers to design and evaluate their network, especially when new providers enter into the same service area, which is missing in the literature.

The main contributions of this study are to model the interference for LPWANs in uplink and to investigate the impact of having several coexisting technologies on the performance metrics such as expected number of required retransmissions and mean transmissions success probability. In particular, we consider a LPWAN scenario, where multiple technologies coexist over a set of shared radio resources in the same area, and investigate statistics of reliability of communications (for each of the coexisting technologies). Towards this end, first we derive a model of interference for asynchronous short-packet communications, from which, the transmission success probability and its moments are derived subsequently. Furthermore, the expected number of transmission attempts to successfully transmit a packet, known as the mean local delay [10], is derived by utilizing the beta distribution and leveraging an approximate ${ }^{1}$ yet tractable expression of meta distribution. Mean local delay lower bounds different notions of delay such as single-hop and end-to-end delays, and can measure the interference correlation in network with multiple sources of interference. Infinite mean local delay denotes a little hope for network to provide any useful service to its users. Thus, it is a fundamental and fine-grained metric to study [13]. Finally, we leverage the derived expressions and investigate stability of the network, i.e., figure out boundary values for deployment and operation parameters (like access point density and traffic load) for which, mean local delay is finite. These derivations provide a framework for studying distribution of QoS in LPWANs instead of the average values.

\section{SYSTEM MODEL}

We consider uplink transmissions in an IoT network with massive number of IoT devices and several access points, as shown in Fig.1. The IoT devices belong to $\mathcal{K}$ different data transmission protocols, where each protocol utilizes a distinct transmission pattern ${ }^{2}$. Each transmission pattern is characterized by three parameters: the time/bandwidth of transmission, the transmit power, and the transmission intervals. Due to the distinct transmission patterns of protocols, transmitted packets might have partial overlapping in time and frequency. From now on, we refer to devices belonging to data transmission protocol $j \in\{1 \ldots \mathcal{K}\}$ as class- $j$ devices. Devices belonging to the class- $j$ are randomly distributed using Poisson point process with parameters $\lambda_{j}$. The average time between two consecutive transmissions, the average transmission time of a

\footnotetext{
${ }^{1}$ The accuracy of such an approximation has been investigated in [10].

${ }^{2}$ Different protocols may refer to different technologies or different usecases of a single technology. The information about $\mathcal{K}$ and the number of active devices can be acquired by network monitoring or by exchanging of the information between operators
}

packet, and the bandwidth of transmissions for class- $j$ devices are denoted by $T_{r_{j}}$ seconds, $T_{p_{j}}$ seconds, and $\omega_{j}$ respectively. Devices transmit their data to their closest APs which are distributed in the network with Poisson point process ${ }^{3}$ with density of $\lambda_{j}^{\mathrm{AP}}$ referring to the distribution of APs serving class- $j$ devices. The channel coefficients consist of path loss and the Rayleigh fading components. When a packet is triggered at a device, it is transmitted immediately within the shared frequency resources, i.e. in the range of $\left(f_{\min }, f_{\max }\right)$. Denote the total uplink interference on the AP serving class- $j$ devices by $I_{j}$ which is calculated as, $I_{j}=\sum_{i=1}^{\mathcal{K}} I_{i, j} . I_{i, j}$ is the interference from class- $i$ active devices on the AP serving class- $j$ devices and can be expressed as,

$$
I_{i, j}=\sum_{\phi_{i}} h_{i, j} L_{i, j}(d) \nu_{i} P_{i} .
$$

In this equation, $\phi_{i}$ denotes the set of active class- $i$ devices that contribute to the interference, $h_{i, j}, L_{i, j}(d)=\left\|d_{i, j}\right\|^{-\alpha}$, and $P_{i}$ are Rayleigh fading, pathloss function with $d$ as the distance from the device to the corresponding AP, and transmission power of class- $i$ devices, respectively. $\nu_{i}$ is the time-frequency activity of class- $i$ transmissions over shared resources. The activity factor is proportional to the ratio of utilized bandwidth to the total available bandwidth. The higher $\omega_{i}$ is, the higher $\nu_{i}$ is and hence more interference might be created for other transmission protocols. $\nu_{i}$ is calculated as,

$$
\nu_{i}=\frac{T_{p_{i}}}{T_{r_{i}}} \frac{\omega_{i}}{f_{\max }-f_{\min }} .
$$

In this expression, $\nu_{i}$ gets its maximum value $\left(\nu_{i}=1\right)$ at $T_{p_{i}}=$ $T_{r_{i}}$, and $\omega_{i}=f_{\max }-f_{\min }$, i.e., when transmission from class- $i$ spans over all time-frequency resources. The SINR of a class$j$ device, indexed by $m$, is given by $\gamma_{j, m}=\frac{P_{j} h_{m, j} L_{m, j}(d)}{\mathcal{N}+I_{j}}$, where $\mathcal{N}$ is the noise power.

\section{ThEORETICAL ANALYSIS}

In this section, we derive the success probability and $b$-th moment of success probability in order to approximate the meta distribution, and derive the mean local delay. We define the success probability, as the probability of having SINR higher than a threshold, i.e., $\gamma_{\mathrm{th}}$, conditioned on the activity of the target device, denoted by $t x$ in (3) and point processes of other devices defined by the set $\phi_{i}$. The success probability is derived as follows,

$$
P_{\mathrm{sc}, j}\left(\gamma_{\mathrm{th}}\right) \triangleq \mathcal{P}\left(\gamma_{j} \geq \gamma_{\mathrm{th}} \mid \phi ; t x\right)
$$

In the following we derive the meta distribution and mean local delay based on definition of the $P_{\mathrm{sc}, j}$.

\section{A. Meta Distribution}

The meta distribution of the SINR, i.e., $\bar{F}_{P_{\mathrm{sc}, j}}$, is defined as the complementary cumulative distribution function (CCDF) of the random variable $P_{\mathrm{sc}, j}$ which is given by,

$$
\bar{F}_{P_{\mathrm{sc}, j}} \triangleq \mathbb{P}^{0}\left(P_{\mathrm{sc}, j}\left(\gamma_{\mathrm{th}}\right) \geq x\right), \quad x \in[0,1]
$$

${ }^{3}$ The justification of modelling AP locations with PPP is explained in [14]. 


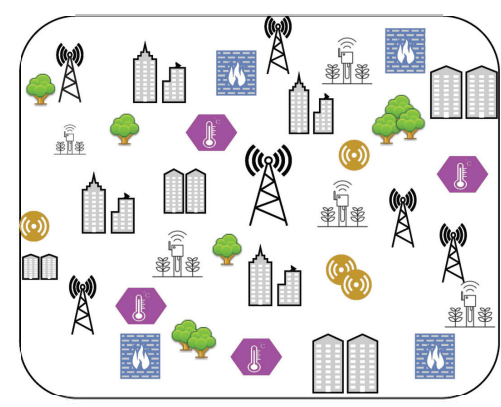

(a) Spatial model

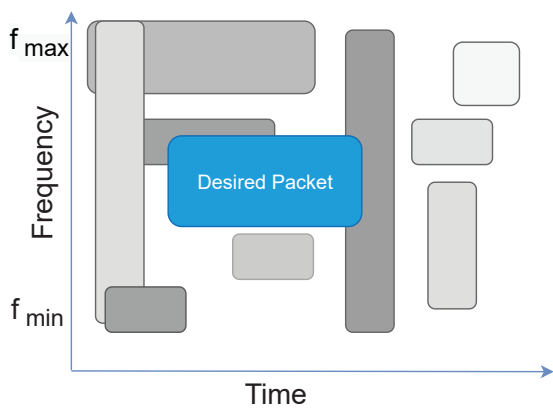

(b) Interference model

Fig. 1: Spatial and interference model

In (4), $\mathbb{P}$ is the Palm measure ${ }^{4}$ conditioning on the investigated AP located at the origin 0 and on the corresponding transmitter to be active. Meta distribution is the probability that the random node locations are such that the link under consideration has a reliability of $x$ or higher. Since all point processes in the model are ergodic, the meta distribution can be interpreted as the fraction of the active links whose conditional success probabilities are greater than $x$. In other words, it provides fine-grained information on the SIR/SINR and answers questions such as "What fraction of links in a network achieve $x \%$ link reliability if the required SIR is $\gamma_{\text {th }}$ dB?" [9].

One way of calculating the meta distribution is to derive the imaginary moments of transmission success probability, i.e., $M_{j t}, j=\sqrt{-1}, t \in \mathbb{R}$ and apply the Gil-Pelaez theorem. A simpler approach is to approximate the meta distribution with the beta distribution [10]. The advantage of the latter approach is that it requires only the first and second moments of $P_{\mathrm{sc}, j}$. The meta distribution approximated by the beta approximation is derived as follows,

$$
\bar{F}_{P_{\mathrm{sc}, j}} \approx 1-I_{x}\left(\frac{\beta M_{1}}{1+\beta M_{1}}, \beta\right) \quad x \in[0,1]
$$

where $\beta \triangleq \frac{\left(M_{1}-M_{2}\right)\left(1-M_{1}\right)}{M_{2}-M_{1}^{2}}, M_{1}$ and $M_{2}$ are the first and second moments of $P_{\mathrm{sc}, j}, I_{x}(a, b)$ is the regularized incomplete beta function $I_{x}(a, b)=\frac{\int_{0}^{x} t^{a-1}(1-t)^{b-1} d t}{B(a, b)}$ and $B(a, b)$ denotes the beta function.

To calculate (5) for class- $j$ devices, we need to derive $P_{\mathrm{sc}, j}$, and then the $b$-th moment of $P_{\mathrm{sc}, j}\left(\gamma_{\mathrm{th}}\right)$, i.e., $M_{b}\left(P_{\mathrm{sc}, j}\left(\gamma_{\mathrm{th}}\right)\right)$.

\footnotetext{
${ }^{4}$ The Palm measure is the conditional point process distribution given that a point (the investigated AP) exists at a specific location [15].
}

We omit the subscript $j$ for simplicity. The success probability is given by,

$$
\begin{aligned}
& P_{s c}\left(\gamma_{\mathrm{th}}\right)=\mathcal{P}\left(\gamma \geq \gamma_{\mathrm{th}} \mid \phi ; \mathrm{tx}\right) \\
& =\mathcal{P}\left(h \geq \frac{\gamma_{\mathrm{th}}\left(\mathcal{N}+I_{j}\right)}{P_{j} L(d)} \mid \phi ; \mathrm{tx}\right) \\
& \stackrel{(a)}{=} P_{N, 1} E_{h}\left[\exp \left(-\gamma_{\mathrm{th}} d^{\alpha}\left[\sum_{i=1}^{\mathcal{K}} \frac{P_{i}}{P_{j}} \sum_{\phi_{i}} h_{i, j}\left\|d_{i, j}\right\|^{-\alpha} \nu_{i, j}\right]\right)\right] \\
& \stackrel{(b)}{=} P_{N, 1} \prod_{i=1}^{\mathcal{K}}\left[\prod_{d_{i, j} \in \phi_{i}}\left(\frac{p_{i}}{1+\gamma_{\mathrm{th}} d^{\alpha} \frac{P_{i}}{P_{j}} \nu_{i, j}\left\|d_{i, j}\right\|^{-\alpha}}+1-p_{i}\right)\right] .
\end{aligned}
$$

In above equations, (a) follows from the unit mean exponential distribution of the channels gains, and (b) is obtained by deriving the expectation with respect to the channel gains and channel access scheme. $P_{N, 1}$ is the expected value of thermal noise and can be neglected in interference limited networks [9]. $p_{i}$ is the probability of class $i$ device being active and $\alpha$ is the pathloss exponent. Let $P_{N, b}=E\left(P_{s c}^{b}\left(\mathrm{SNR} \geq \gamma_{\mathrm{th}}\right)\right)=$ $\exp \left(-\frac{\gamma_{\mathrm{th}} \mathcal{N} b d^{\alpha}}{P_{j}}\right)$ be the impact of noise on the $b$-th moment of $P_{s c}$. Let us consider an interference-limited scenario and remove the term corresponding to noise $\mathrm{s}^{5}$. The $b$-th moment of the $P_{\mathrm{sc}}\left(\gamma_{\mathrm{th}}\right)$ is calculated as follows,

$M_{b}=$

$E_{d}\left(\prod_{i=1}^{\mathcal{K}}\left[\prod_{d_{i, j} \in \phi_{i}}\left(\frac{p_{i}}{1+\gamma_{\mathrm{th}} d^{\alpha} \frac{P_{i}}{P_{j}} \nu_{i, j}\left\|d_{i, j}\right\|^{-\alpha}}+1-p_{i}\right)^{b}\right]\right)$

$\stackrel{(a)}{=}$

$E_{d}\left(\prod_{i=1}^{\mathcal{K}} \exp \left(\int_{\mathbb{R}^{2}}-\lambda_{i}\left[1-\left(\frac{p_{i}}{1+\gamma_{\mathrm{h}} d^{\alpha} \frac{P_{i}}{P_{j}} \nu_{i, j}\left\|d_{i, j}\right\|^{-\alpha}}+1-p_{i}\right)^{b}\right] d x\right)\right)$

$\stackrel{(b)}{=}$

$E_{d}\left(\exp \left(-\sum_{i=1}^{\mathcal{K}} \lambda_{i} \pi\left(\gamma_{\mathrm{th}} \frac{P_{i}}{P_{j}} \nu_{i, j}\right)^{\delta} d^{2}\left(\frac{\pi \delta}{\sin \pi \delta}\right) \sum_{m=1}^{\infty}\left(\begin{array}{c}b \\ m\end{array}\right)\left(\begin{array}{c}\delta-1 \\ m-1\end{array}\right) p_{i}^{m}\right)\right)$

where (a) follows from Slivnyak's theorem and steps of deriving (b) is explained in [9]. The expression in (7) is derived for a given distance. Therefore, we take expectation over distance $d$ with distribution given by [11],

$$
f_{D}(d)=\frac{5}{2} \pi \lambda_{j}^{\mathrm{AP}} d \exp \left(-\frac{5}{4} \pi \lambda_{j}^{\mathrm{AP}} d^{2}\right) .
$$

$D_{b}(p, \delta)$ can be defined as,

$$
\begin{aligned}
D_{b}(p, \delta) & =\sum_{m=1}^{\infty}\left(\begin{array}{c}
b \\
m
\end{array}\right)\left(\begin{array}{c}
\delta-1 \\
m-1
\end{array}\right) p_{i}^{m} \\
& =p b_{2} F_{1}(1-b ; 1-\delta ; p) \quad \text { for } \quad b \in \mathbb{C}, p, \delta \in[0,1]
\end{aligned}
$$

where ${ }_{2} F_{1}(a ; b ; p)$ is the hypergeometric function which enables calculating the combinations with non integer values and $\delta=\frac{2}{\alpha}$, where $\alpha$ is pathloss exponent [9]. Then, $M_{b}$ can be

\footnotetext{
${ }^{5}$ Extending the results to noise/interference-limited scenarios is straightforward as noise simply adds a constant term to the analytical results [10].
} 
calculated by taking the expectation with the given distribution of distance between devices and the AP as follows:

$$
\begin{aligned}
& M_{b}= \\
& \int_{0}^{\infty} \exp \left(-\left(\sum_{i=1}^{\mathcal{K}} \lambda_{i} \pi\left(\gamma_{\mathrm{th}} \frac{P_{i}}{P_{j}} \nu_{i, j}\right)^{\delta} \frac{\pi \delta}{\sin \pi \delta} D_{b}(p, \delta)\right) r^{2}\right) f_{D}(r) d r \\
& =\left[\frac{4}{5 \pi} \sum_{i=1}^{\mathcal{K}} \frac{\lambda_{i}}{\lambda_{j}^{\mathrm{AP}}} \pi\left(\gamma_{\mathrm{th}} \frac{P_{i}}{P_{j}} \nu_{i, j}\right)^{\delta}\left(\frac{\pi \delta}{\sin \pi \delta}\right) D_{b}(p, \delta)+1\right]^{-1} \text {. (10) }
\end{aligned}
$$

For $b \in \mathbb{N}$, the infinite sum in (10) becomes finite and has only $\mathrm{b}$ terms.

Each moment has its own interpretation in the network. For instance, $M_{-1}$ is the mean local delay or expected number of retransmissions to successfully transmit a packet. $M_{1}$ is the mean transmission success probability. The second moment, $M_{2}$, is the mean-squared value of transmission success probability. This value is used to derive the variance (or standard deviation of transmission success probability) i.e., Variance $=M_{2}-M_{1}^{2}$. The variance measures the dispersion of a link's $P_{s}$ relative to the mean success probability. The first and second moment can be simply calculated by setting $b=1,2$ in (10), respectively.

$$
\begin{aligned}
& M_{1}=\text { Mean success probability }= \\
& {\left[\frac{4}{5 \pi} \sum_{i=1}^{\mathcal{K}} \frac{\lambda_{i}}{\lambda_{j}^{\mathrm{AP}}} \pi\left(\gamma_{\text {th }} \frac{P_{i}}{P_{j}} \nu_{i, j}\right)^{\delta}\left(\frac{\pi \delta}{\sin \pi \delta}\right)_{2} F_{1}\left(0 ; 1-\delta ; p_{i}\right)+1\right]^{-1}}
\end{aligned}
$$

$M_{2}=$

$\left[\frac{4}{5 \pi} \sum_{i=1}^{\mathcal{K}} \frac{\lambda_{i}}{\lambda_{j}^{\mathrm{AP}}} \pi\left(\gamma_{\mathrm{th}} \frac{P_{i}}{P_{j}} \nu_{i, j}\right)^{\delta}\left(\frac{\pi \delta}{\sin \pi \delta}\right)_{2} F_{1}\left(-1 ; 1-\delta ; p_{i}\right)+1\right]^{-1}$

Mean success probability and the variance in (11) and (12) can be adjusted (for instance when a new set of interferers is deployed in the area, i.e. $\mathcal{K}$ is increased), by adjusting the transmission power (for devices with power control property), the activity factor $\left(\nu_{j}\right)$, probability of being active $\left(p_{j}\right)$, or the density of APs.

\section{B. Mean Local Delay}

Mean local delay (MLD) is defined as the expected number of (re)transmission attempts, denoted by $N^{t x}$, which is required for a device to successfully transmit a packet to its AP. MLD can be derived as follows,

$$
\mathbb{E}\left(N^{t x}\right)=\mathbb{E}\left(\mathbb{E}\left(N^{t x} \mid \Phi\right)\right) \stackrel{(a)}{=} \mathbb{E}\left(\frac{1}{P_{s c}}\right)=M_{-1} .
$$

In the above equation, the inner expectation is obtained by averaging over the fading and the ALOHA. (a) in (13) is obtained since, conditioned on $\Phi, N^{t x}$ is geometrically distributed with parameter $P_{s c}$, i.e., letting $N_{\Phi}^{t x}=\left(N^{t x} \mid \Phi\right)$, we have $\mathbb{P}\left(N_{\Phi}^{t x}=k\right)=\left(1-P_{\mathrm{sc}}\right)^{k-1} P_{\mathrm{sc}}, k \in \mathbb{N}$. Let $\zeta=\left(1-p_{i}\right)^{(\delta-1)}$, we can derive $M_{-1}$ using the following expression, $M_{-1}=M_{1}^{-\zeta}$ [9]. In (7), let $b=-1$ and derive the mean value of $M_{-1}$ based on the distance distribution defined in (8), then the MLD is derived as,

$$
\begin{aligned}
& M_{-1}= \\
& {\left[1-\frac{4}{5 \pi}\left(\sum_{i=1}^{\mathcal{K}} \frac{\lambda_{i}}{\lambda_{j}^{\mathrm{AP}}} \pi\left(\gamma_{\mathrm{th}} \frac{P_{i}}{P_{j}} \nu_{i, j}\right)^{\delta}\left(\frac{\pi \delta}{\sin \pi \delta}\right)_{2} F_{1}\left(2 ; 1-\delta ; p_{i}\right)\right) \zeta\right]}
\end{aligned}
$$$$
\text { (14) }
$$

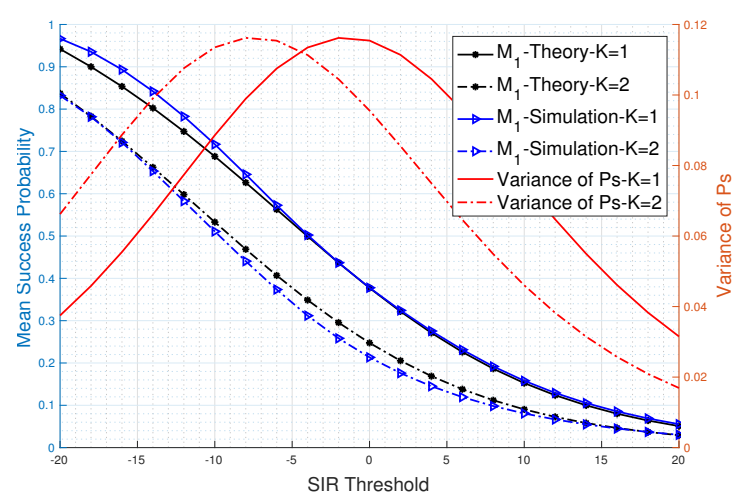

Fig. 2: Mean/variance of $P_{\mathrm{sc}}$ with $p_{i}=0.5$.

The expression in (14) is valid when the denominator is positive. This constraint defines a region in which MLD is finite. According to (14), increasing the devices density and $p_{i}$, will increase the MLD to the point it tends to infinity. Infinite MLD means there exists at least one link in the network in which even with multiple transmissions it is not possible to satisfy the SINR requirement. Another interesting result is the case when the density of devices belonging to one protocol (or time-frequency activity) increases. The degradation due to this increase can be compensated by adjusting the transmit power of the devices, if it is possible, or by adjusting the density of serving APs.

\section{Performance Evaluations}

\section{A. Validation of Analytical Results}

In this section, we validate the analytical results with simulations and investigate the impact of the offered load, AP density, and SIR threshold on the meta distribution, success probability, and mean local delay. We assume that there are 10 active APs in the service area and devices are randomly distributed using Poisson point process with parameters $\lambda_{i}$ over area of $400 \mathrm{~km}^{2}$. We set the transmission power of devices to $20 \mathrm{~mW}$ and assume that this transmission power is similar for all transmission protocols. We set the $\lambda_{i}=10^{-3} \mathrm{~m}^{-2}$, $p_{i}=0.5, \alpha=4, T_{p_{i}}=10 \mathrm{~ms}, T_{r_{i}}=2 \mathrm{~ms}, \omega_{i}=100 \mathrm{KHz}$, and $f_{\max }-f_{\min }=200 \mathrm{KHz}$. In Fig. 2 , we compare the derived expression for average success probability with the simulation. We plot the variance of success probability which represents the variation of reliability at given SIR threshold. Confirmed by Fig. 2, more variations on reliability is expected at lower SIR threshold as more number of technologies coexist in the network. When $\mathcal{K}$ is larger, more interference is created in the network. To keep the $P_{s c}$ fixed, $\gamma_{t h}$ should be lower. This means a shift to the left for curves such as variance.

\section{B. Reliability and scalability evaluations}

In Fig. 3, we investigate the impact of $p_{i}$ which is the offered load and is defined as the time-frequency activity of a device from protocol $i$. It can be seen that for each SIR threshold there is a load limit which after that the MLD, derived in (14), becomes infinite. This means that there exists at least one device such that the transmission problem cannot be resolved 


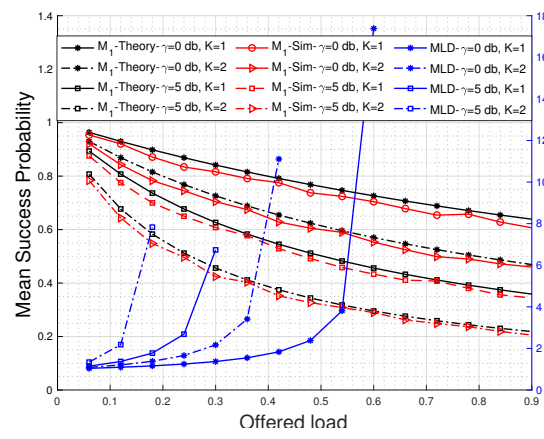

Fig. 3: MLD and offered load

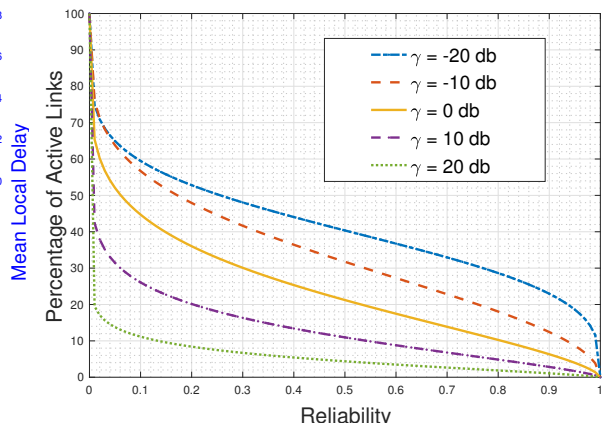

Fig. 4: Meta distribution of $P_{\mathrm{sc}}$ for $p_{i}=0.5$.

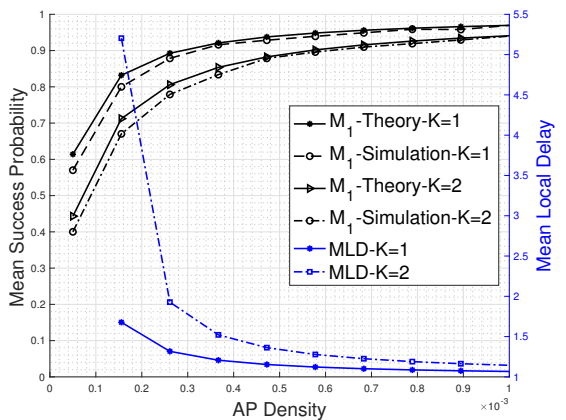

Fig. 5: MLD and $P_{\mathrm{sc}}$ with $p_{i}=0.5$. by retransmission. By decreasing $p_{i}$, the network load is lower and hence less number of retransmissions is required. On the other hand, increasing $p_{i}$, results in decreasing the success probability, due to traffic congestion in the network.

In Fig. 4, $Y$-axis shows the percentage of links in the network that have the SIR greater than the SIR threshold with the reliability threshold defined in $X$-axis, which is derived in (5). The higher the reliability requirement is, the less number of links can provide such a reliability.

Fig. 5 investigates the impact of APs density on the meta distribution, MLD, and the mean success probability. Fig. 5 shows that decreasing the AP density will make the MLD infinite while increasing the AP density does not have much impact on the number of transmission attempts (as it is very close to 1). On the other hand, increasing the AP density will increase both the success probability and the reliability of the links. However, this improvement will not have great impact after a point on the mean success probability and mean local delay. This observation suggests that resource provisioning for this type of network is critical since providing resources will increase the performance metrics, however, there is a limit on the number of APs after which the network can not benefit from deploying more APs.

\section{CONCLUSION}

In this letter, we have investigated reliability and latency performance of LPWANs in coexistence scenarios, where IoT devices utilize different data transmission protocols for data transmission over shared resources. Leveraging tools from stochastic geometry, we have derived a closed-form expression for the expected reliability of communications, distribution of reliability, and the expected number of packet transmissions (known as mean local delay) fo a successful packet transmission. We have further investigated the impact of traffic load of devices and density of APs on the meta distribution of success probability and mean local delay. The analysis of mean local delay enables us to infer existence of unreliable links, despite a high average success probability across the network, and hence have a broader vision of coverage over the service area. Since infinite mean local delay (or higher than the data expiration deadline) corresponds to a situation in which network cannot provide any useful services to the users, we derive the regions of communication parameters; including AP densities, time-frequency occupation of services' communications, and transmit powers of devices; that result in a finite mean local delay. These results enable service providers to orchestrate and operate their networks when other service providers coexist in their service area, and are sharing the radio resources.

\section{REFERENCES}

[1] R.B. Sørensen et al., "Analysis of latency and MAC-layer performance for class a LoRaWAN," IEEE Wireless Communications Letters, vol. 6, no. 5, pp. 566-569, 2017.

[2] O. Georgiou and U. Raza, "Low power wide area network analysis: Can LoRa scale?" IEEE Wireless Communications Letters, vol. 6, no. 2, pp. 162-165, 2017.

[3] Z. Li et al., "2D time-frequency interference modelling using stochastic geometry for performance evaluation in low-power wide-area networks," in IEEE ICC, 2017.

[4] R. Abbas et al., "A novel analytical framework for massive grant-free NOMA," IEEE Transactions on Communications, vol. 67, no. 3, pp. 2436-2449, 2018.

[5] T. Elshabrawy and J. Robert, "Closed-form approximation of LoRa modulation BER performance," IEEE Communications Letters, vol. 22, no. 9, pp. 1778-1781, 2018.

[6] M. Lauridsen et al., "Interference measurements in the european 868 MHz ISM band with focus on LoRa and SigFox," in IEEE WCNC, 2017.

[7] Z. Qin et al., "Modelling and analysis of low-power wide-area networks," in 2017 IEEE International Conference on Communications (ICC). IEEE, 2017, pp. 1-7.

[8] M. Masoudi et al., "Grant-free radio access IoT networks: Scalability analysis in coexistence scenarios," in IEEE ICC. IEEE, 2018, pp. 1-7.

[9] M. Haenggi, "The meta distribution of the SIR in poisson bipolar and cellular networks," IEEE Transactions on Wireless Communications, vol. 15, no. 4, pp. 2577-2589, 2015.

[10] M. Salehi et al., "Analysis of D2D underlaid cellular networks: SIR meta distribution and mean local delay," IEEE Transactions on Communications, vol. 65, no. 7, pp. 2904-2916, 2017.

[11] _ - "Meta distribution of SIR in large-scale uplink and downlink NOMA networks," IEEE Transactions on Communications, vol. 67, no. 4, pp. 3009-3025, 2018.

[12] N. Zabetian et al., "Energy-efficient power allocation for device-todevice communications underlaid cellular networks using stochastic geometry," Transactions on Emerging Telecommunications Technologies, vol. 30, no. 12, p. e3768, 2019.

[13] M. Haenggi, "The local delay in poisson networks," IEEE Transactions on Information Theory, vol. 59, no. 3, pp. 1788-1802, 2012.

[14] J.G. Andrews et al., "A primer on spatial modeling and analysis in wireless networks," IEEE Communications Magazine, vol. 48, no. 11, pp. 156-163, 2010.

[15] M. Haenggi, Stochastic geometry for wireless networks. Cambridge University Press, 2012. 